\title{
Nimotuzumab combined with radiotherapy for esophageal cancer: preliminary study of a Phase II clinical trial
}

\author{
This article was published in the following Dove Press journal: \\ OncoTargets and Therapy \\ 5 November 2013 \\ Number of times this article has been viewed
}

Objective: To determine the safety and therapeutic effects of nimotuzumab (h-R3) combined with radiotherapy in esophageal cancer.

Methods: This Phase II clinical trial involved 42 patients with stage II (inoperable or refused surgery) to stage IV (supraclavicular lymph node metastasis only) esophageal cancers treated between November 2008 and July 2010. All patients had squamous cell carcinomas, and all received three-dimensional conformal radiotherapy and $200 \mathrm{mg}$ nimotuzumab per week during radiotherapy.

Results: There were 9, 25, and 8 patients with stage II, III and IV disease, respectively. All except two patients received 50-70 Gy radiation; 37 patients $(88.1 \%)$ received more than five nimotuzumab doses. Grade III toxicities (21.4\% of all adverse events) included esophagitis and gastrointestinal, dermatological and hematological toxicities. Complete response, partial response, stable disease, and progressive disease were observed in 0, $22(52.4 \%), 17(40.5 \%)$ and $3(7.1 \%)$ patients at 1 month after the treatment. The epidermal growth factor receptor (EGFR) overexpression rate was $95.2 \%$. After a median follow-up of 37 months, the median survival time (MST) was 14 months. The 2 year and 3 year overall survival (OS) rates were $33.3 \%$ and $26.2 \%$, respectively. The median progression-free survival (PFS) time was 10 months. The 2 year and 3 year PFS rates were $24.5 \%$ and $22.1 \%$, respectively. The MST in the 13 patients with (+++) EGFR expression (group A) and 7 patients with (++) EGFR expression (group B) was 15 and 11 months, respectively. The 2 year and 3 year OS rates were $46.2 \%$ and $38.5 \%$ in group A and $28.6 \%$ and $28.6 \%$ in group $\mathrm{B}$, respectively $(P=0.405)$.

Conclusion: Although concurrent chemoradiotherapy was the standard care for locally advanced esophageal cancer, radiotherapy was the choice for those who were refused or could not tolerate chemoradiotherapy. Our study shows that nimotuzumab combined with radiotherapy was well tolerated in patients with esophageal cancer. EGFR overexpression was more common than previously reported. OS was higher after combined therapy than after historical control radiotherapy alone. Further studies are required to confirm the therapeutic efficacy of nimotuzumab in esophageal cancer.

Keywords: esophageal neoplasms, nimotuzumab, radiotherapy, targeted therapy, treatment outcomes

\section{Introduction}

Esophageal cancer is the sixth leading cause of cancer-related deaths in the world. ${ }^{1,2}$ The morbidity and mortality rates of esophageal cancer in the People's Republic of China are the highest in the world, and over $50 \%$ of patients have locally advanced or metastatic disease at presentation. ${ }^{3}$ Currently, patients who are unfit to undergo surgery or have locally advanced esophageal cancer are offered concurrent chemoradiotherapy. ${ }^{4-6}$ 
Modern approaches to cancer treatment are focused on combination strategies involving surgery, chemotherapy, radiotherapy, new chemotherapy drugs and targeted therapy.

Many malignant tumors overexpress epidermal growth factor receptor (EGFR), ${ }^{7}$ which initiates signal transduction by activating a receptor-associated tyrosine kinase. EGFR overexpression has been associated with cancer cell invasion, metastasis, and poor prognosis. ${ }^{8}$ EGFR inhibitors have proven efficacious in patients with non-small cell lung, colon, intestinal, and head and neck cancers. ${ }^{9-11}$

Nimotuzumab (h-R3) is a humanized monoclonal antibody. In preclinical studies, nimotuzumab has shown significant antitumor, proapoptotic and antiangiogenic activities. ${ }^{12}$ In an in vitro study, nimotuzumab was shown to radiosensitize a non-small cell lung cancer cell line. ${ }^{13}$ In another, more recent study, nimotuzumab enhanced the effects of radiotherapy on esophageal squamous cell carcinoma cells with a functional and active EGFR pathway. ${ }^{14}$ Several Phase I and II clinical studies have confirmed the safety profile of the combination of nimotuzumab with radiotherapy. Nimotuzumab combined with radiotherapy or chemoradiotherapy has proven clinically beneficial, improving overall survival (OS) and progression-free survival (PFS) in patients with head and neck cancer. ${ }^{15-17}$

Between November 2008 and July 2010, we conducted a Phase II clinical trial in which patients with stage II (inoperable or refused surgery) to stage IV (supraclavicular lymph node metastasis only) esophageal cancer received nimotuzumab in combination with radiotherapy. Most of the patients in our study had previously refused chemotherapy, and others could not tolerate chemotherapy. The purpose of this multicenter, Phase II study was to determine the safety and therapeutic effect of nimotuzumab in combination with radiotherapy in patients with esophageal cancer.

The trial was approved by the China State Food and Drug Administration (CFDA). The study protocol was approved by the ethical review committee, and permission to conduct the study was granted by the institutional review board. The trial has been registered according to Good Clinical Practice at the Cancer Institute and Hospital, Chinese Academy of Medical Sciences (CAMS). All enrolled patients in the trial voluntarily signed the informed consent forms.

\section{Methods}

\section{Endpoints}

The endpoints were toxicity, clinical response, PFS, and OS.

\section{Patient selection and enrollment}

In total, 120 esophageal cancer patients from all across the People's Republic of China were screened. Of these, 42 patients with histologically confirmed stage II (inoperable or refused surgery) to stage IV (supraclavicular lymph node metastasis only) cancer were eligible and were assigned to this trial, which was conducted in six hospitals (Cancer Hospital, CAMS; Cancer Hospital, Harbin Medical University; Tongji Cancer Center Hospital; Cancer Hospital, Tianjin Medical University; Liao-Ning Province Cancer Hospital; Beijing Hospital) between November 2008 and July 2010.

The inclusion criteria were as follows: age, 18-75 years; thoracic-segment esophageal cancer in stages II (inoperable or refused surgery) to IV (supraclavicular lymph node metastasis only); no history of surgery, although history of radio- or chemotherapy was acceptable; estimated survival time, $\geq 3$ months; Karnofsky performance score, $>60$; no serious disease of major organs that would affect physical function; and voluntary signing of consent forms.

The exclusion criteria were as follows: pregnancy or lactation, history of other malignant disease, and joining another clinical trial prior to the study. Of the 42 eligible patients, 34 were male and 8 were female; their median age was 63 years (range, 35-74 years). Table 1 shows further patient characteristics.

All eligible patients underwent endoscopy, biopsy, and computed tomography (CT) of the neck, chest, and abdomen, esophagography, and other physical examinations, including blood tests. Patients with metastases, involvement of more than one lymph node, and lymph nodes $\geq 0.5 \mathrm{~cm}$ in diameter were eligible. Radionuclide bone scans and brain magnetic resonance imaging were offered to the patients. Other tests, such as endoesophageal ultrasonography and positron emission tomography-computed tomography (PET-CT) were widely available at the time of the trial. EGFR expression was recommended to be measured in all samples, if possible.

\section{Treatment schedule}

\section{Radiotherapy}

All patients received three-dimensional conformal radiotherapy with 4-10 Mv X-ray accelerators. A CT simulation with a slice thickness of $5 \mathrm{~mm}$ was obtained before irradiation. The gross tumor target volume (GTV) was defined as the primary tumor, and the GTVnd as the enlarged regional lymph nodes. The clinical target volume (CTV) was defined as the GTV plus an area bounded by a margin of $3 \mathrm{~cm}$ in the superior and inferior directions to the GTV, $6 \mathrm{~mm}$ in the left 
Table I Baseline characteristics of the patients

\begin{tabular}{ll}
\hline & Number of patients \\
\hline Male:female & $34: 8$ \\
Age & Median age 63 (45-74) \\
Pathological type & Squamous cell carcinoma \\
Primary tumor location & \\
Upper thoracic segment & 10 \\
Middle thoracic segment & 26 \\
Lower thoracic segment & 3 \\
Upper and lower thoracic segments & 2 \\
Upper and middle thoracic segments & 1 \\
Stage & \\
II & 9 \\
III & 25 \\
IV & 8 \\
T stage & \\
T2 & 11 \\
T3 & 13 \\
T4 & 18 \\
N stage & \\
No & 13 \\
NI & 29 \\
M stage & \\
M0 & 34 \\
MI & 8 \\
Width of primary tumor $(\mathrm{cm})$ & Median 2.7 $(0.5-5)$ \\
Length of primary tumor $(\mathrm{cm})$ & Median $6(2-15)$ \\
Diameter of lymph node metastasis $(\mathrm{cm})$ & Median I $(0.5-5.5)$ \\
\hline
\end{tabular}

and right directions and $6 \mathrm{~mm}$ in the anterior and posterior directions. The CTV also included the GTVnd and the area of the prophylactic regional lymph node. In the case of uppersegment and middle-segment thoracic esophageal cancers, the superior margin of the prophylactic regional lymph node was the upper border of the $\mathrm{T} 1$ vertebra, and the inferior margin was the subcarinal region. In the case of lower-segment cancers, the superior margin of the prophylactic regional lymph node was the thoracic inlet, and the inferior margin was the left gastric region. The planning target volume (PTV) was defined as the CTV plus an area bounded by a margin of $5 \mathrm{~mm}$ extending three-dimensionally around the CTV. The total dose to $95 \%$ of the PTV was 50-60 Gy, which was administered in 2-Gy once-daily fractions for 5 days a week over 5-6 weeks.

\section{Targeted therapy}

In a clinical trial of the humanized amtiepidermal growth factor receptor monoclonal antibody h-R3 with concurrent chemoradiotherapy for unresectable head and neck carcinomas reported by Crombet et al, it was found that a $200 \mathrm{mg} /$ week of h-R3 (the lab code for nimotuzumab) was the appropriate dose on the basis of h-R3 serum levels and the response and survival. ${ }^{17}$ Therefore, all patients in our trial received 5-6 once-weekly injections of $200 \mathrm{mg}$ h-R3 diluted in $250 \mathrm{~mL} 0.9 \%$ sodium chloride, administered as an intravenous infusion over 1 hour.

\section{Follow-up}

All patients were monitored weekly during the treatment for signs of acute toxicity. Follow-up was conducted at 3 month intervals in the first 3 years and at 6 month intervals thereafter.

\section{Evaluation of therapeutic effect and toxicity}

The endpoints for the trial were toxicity, clinical response, PFS, and OS. Toxic effects were assessed once a week, using the National Cancer Institute's Common Toxicity Criteria, version 3.0. ${ }^{18}$ Clinical response was assessed using the Response evaluation criteria in solid tumors (RECIST) ${ }^{19}$ Twodimensionally measurable disease was defined by the length and width of the primary tumor and the diameter of the largest positive lymph node. Response in assessable disease was determined by at least two observers. OS was defined as the interval between the date of treatment commencement and the date of death from esophageal cancer or treatment-related causes. Patients who died from other causes were excluded from the analysis. The rates of adverse events and toxic effects, and the OS were determined. All case report forms were assessed centrally in the Cancer Hospital, CAMS.

\section{Statistical analysis}

We performed an intention-to-treat analysis. SPSS software, version 11.5 (IBM Corporation, Armonk, NY, USA), was used. Analyses were also conducted for subgroups based on age, gender, performance status, tumor location, pathological type, EGFR expression, and tumor length and width. Survival time was calculated from the date of the first treatment until the date of death. PFS and OS data were estimated with the Kaplan-Meier method, and EGFR expression subgroups were compared using the log-rank test.

\section{Results}

\section{Treatment outcomes}

\section{Toxicity}

The common acute toxicities during the treatment were esophaghitis, and blood/bone marrow, dermatological, and gastrointestinal complications; Table 2 shows the details by grade and incidence. Other toxicities included grade 1 paresthesia (1/42), septicemia (1/42), herpes virus infection (1/42), hyperbilirubinemia (1/42), pulmonary infection (3/42), 
Table 2 Common acute toxicities during treatment

\begin{tabular}{lllll}
\hline & Grade I & Grade 2 & Grade 3 & Grade 4 \\
\hline Esophagitis & $16(38.1 \%)$ & $20(47.6 \%)$ & $3(7.1 \%)$ & 0 \\
Blood/bone marrow & $14(33.3 \%)$ & $10(23.8 \%)$ & $1(2.4 \%)$ & 0 \\
Dermatological & $15(35.7 \%)$ & $4(9.5 \%)$ & $4(9.5 \%)$ & 0 \\
Gastrointestinal & $6(14.3 \%)$ & $2(4.8 \%)$ & $1(2.4 \%)$ & 0 \\
\hline
\end{tabular}

severe fatigue (2/42), fever (9/42) and tracheoesophageal fistula (1/42). Only one patient was allergic to nimotuzumab. The incidence of grade 3 toxicity was $21.4 \%$; no grade 4 toxicity occurred. Mild, nimotuzumab-related skin rash was observed in four patients but did not require treatment.

All patients received 50-60 Gy of irradiation, except for two patients who received only $46 \mathrm{~Gy}$ (one patient refused to continue treatment, and the other patient requested surgery), and one patient who received $70 \mathrm{~Gy}$. In all, 37 (88.1\%) patients received five or six doses of 200-mg nimotuzumab, and five patients received this dose fewer than 5 times because of adverse effects: allergy, pulmonary infection, severe fatigue, or tracheoesophageal fistula.

\section{Therapeutic effects}

The date of the last follow-up was January 30, 2013. The median follow-up time was 37 months (range $31-41$ months). At 1 month after the treatment, none of the patients had attained complete response (CR); $22(52.4 \%)$ patients had achieved partial response (PR); whereas 17 (40.5\%) and $3(7.1 \%)$ patients showed stable disease (SD) and progressive disease (PD), respectively. The objective clinical response was evaluated at the last follow-up, and CR, PR, SD, and PD were observed in 7 (16.7\%), 3 (7.1\%), 0 (0\%), and $32(76.2 \%)$ patients, respectively. Six patients had achieved CR by the sixth month after treatment, and another patient attained CR by the seventh month. The median survival time (MST) was 14 months, and the median PFS was 10 months. The 2 year and 3 year OS rates were $33.3 \%$ and $26.2 \%$, respectively, and the corresponding PFS rates were $24.5 \%$ and $22.1 \%$ (Figure 1). Univariate and multivariate analyses showed that gender, age $\geq 65$ years, stage IV disease, lower-segment tumors, and tumor width and length were not predictors of OS and PFS. Local recurrence and distant metastases were observed in $20(47.6 \%)$ and 14 (33.3\%) patients, respectively. Twelve metastases were detected in the lungs, three in the bones, three in the liver, two in the pleura, one in the hypodermis, and one in a distant lymph node. At the last follow-up, 31 (73.8\%) patients had died: $16(38.1 \%)$ of local recurrence, 13 (31\%) of metastases, and 2 (4.8\%) of other causes. The median disease-specific survival time was

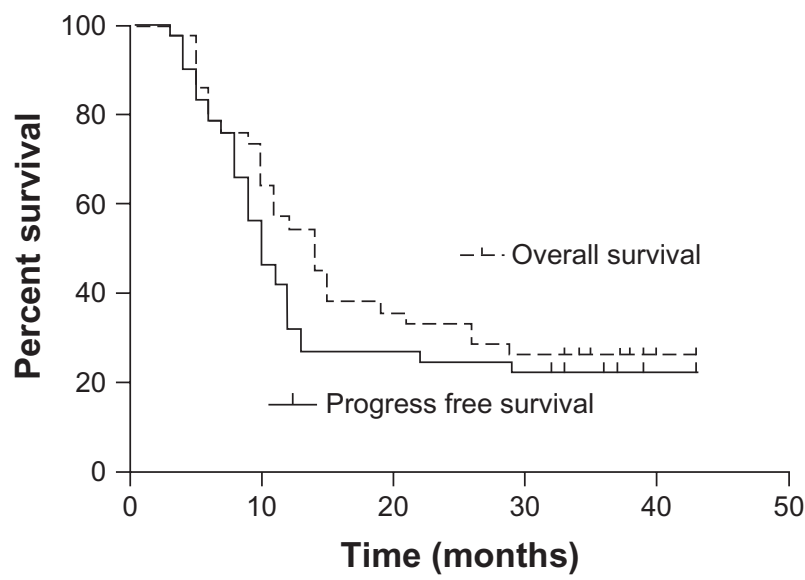

Figure I Overall survival rate and progression-free survival rate of 42 patients with esophageal squamous cell carcinoma.

14 months. The 2 year and 3 year disease-specific survival rates were $32.7 \%$ and $32.7 \%$, respectively.

\section{EGFR expression}

EGFR expression was determined in 21 patients; biopsy specimens were obtained from these patients via esophagoscopy and subjected to an immunochemical assay. The immunoreactivity of EGFR was graded into four groups according to the intensity of cell membrane EGFR staining in the whole tumor: high $(+++)$, markedly stronger staining than normal esophageal epithelium; medium (++), moderately stronger staining than normal esophageal epithelium; low (+), staining identical to that of normal epithelium (Figure 2); and negative (-), faint staining. Strong (+++) and moderate (++) staining indicated EGFR overexpression, ${ }^{20}$ and were found in 20 patients (95.2\%). EGFR expression at levels (++), (+) and (+) was found in 13, 7, and 1 patient, respectively. The MST in the 13 patients with (++) EGFR expression (group A) and the 7 patients with (++) EGFR expression (group B) was 15 and 11 months, respectively. The 2 year and 3 year OS rates were $46.2 \%$ and $38.5 \%$, respectively, in group A and $28.6 \%$ and $28.6 \%$, respectively, in group B; the between-group differences were not significant $(P=0.405$; Figure 3$)$.

\section{Discussion}

In our study of esophageal cancer patients, nimotuzumab combined with radiotherapy was well tolerated. The incidence of grade 3 toxicity was $21.4 \%$; no grade 4 toxicity occurred. Mild nimotuzumab-related rash occurred, but nimotuzumab-related diarrhea did not occur. Only one patient was allergic to nimotuzumab. Nimotuzumab did not seem to increase the acute toxicity of radiotherapy. Safran et al have reported incidence rates of $23 \%, 15 \%$, and $5 \%$ for skin 


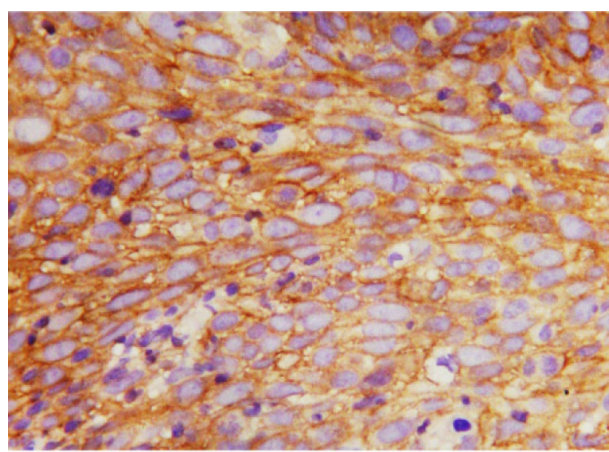

EGFR(+++)

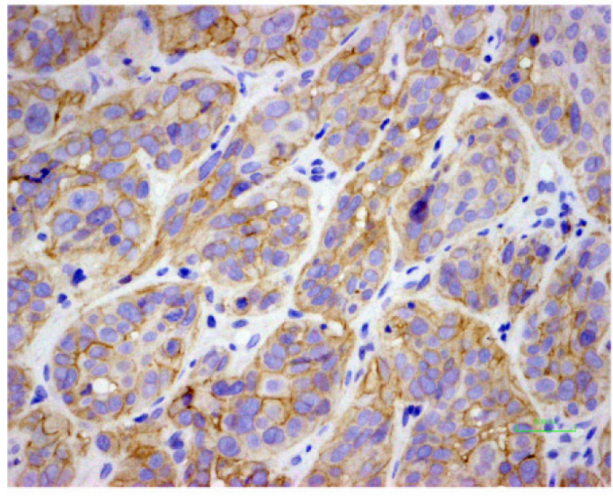

EGFR(++)

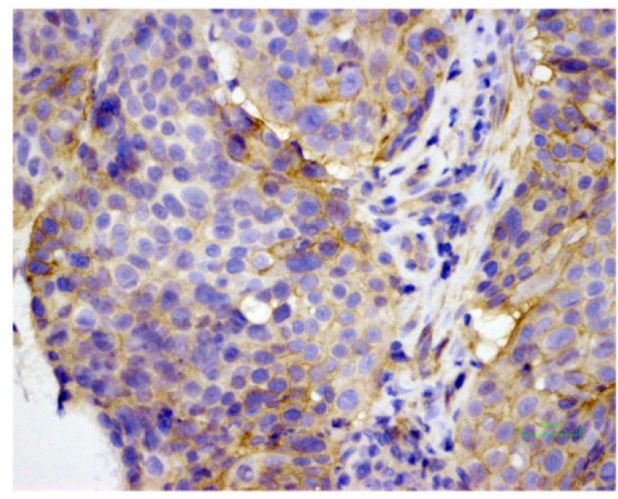

EGFR(+)

Figure 2 The representative pictures of EFGR expression with $(+)$ to $(+++)$ staining.

Abbreviation: EGFR, epidermal growth factor receptor.

rash, esophagitis and hypersensitivity of grades 3 or higher, respectively, in patients receiving HER2-targeted therapy for esophageal tumors. ${ }^{21}$

In our study, most patients who achieved CR did so at 6 months after the treatment, suggesting a delayed therapeutic effect. This finding might indicate that the best time to evaluate the objective clinical response, especially $\mathrm{CR}$, is at 6 months after treatment.

The 2 year and 3 year OS rates were $33.3 \%$ and $26.2 \%$, respectively, and the corresponding PFS rates were $24.5 \%$

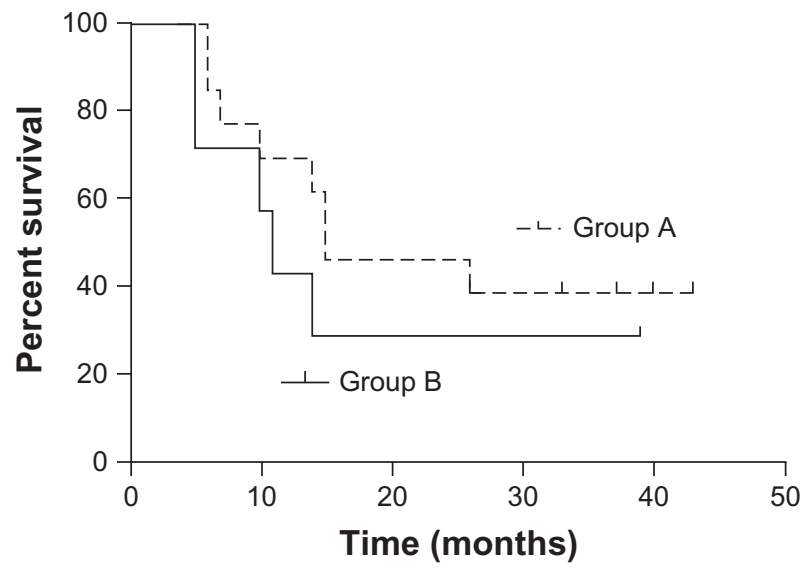

Figure 3 Overall survival rates in patients with $(+++)$ EGFR expression (group $A$, $\mathrm{n}=13$ ) and (++) EGFR expression (group B, $\mathrm{n}=7$ ).

Abbreviation: EGFR, epidermal growth factor receptor.

and $22.1 \%$. The MST was 14 months. These results are more favorable than those reported for radiotherapy alone. In a study comparing concurrent chemoradiotherapy and radiotherapy alone in esophageal cancer patients with lymph node metastasis, the 2 year and 3 year OS rates in the radiotherapy-alone group were $38.5 \%$ and $18.5 \%$, respectively. ${ }^{22}$ In the Radiation Therapy Oncology Group 85-01 trial, no patient was alive in the radiotherapy-alone group after 3 years; the MST in this group was 8.9 months. ${ }^{5}$ In an Eastern Cooperative Oncology Group trial, the MST was 9.2 months and the 3 year OS was $8 \%$ in the radiotherapy-alone group. ${ }^{23}$

For patients with locally advanced esophageal cancer (those who are inoperable or who refuse surgery), concurrent chemoradiotherapy is the standard of care. Most patients in our study refused chemotherapy, and others could not tolerate chemotherapy. For such patients, radiotherapy alone seems to be the only treatment currently accepted/offered. However, our study showed that combined radiotherapy and targeted therapy could improve the OS over that achievable with radiotherapy alone.

The rate of EGFR overexpression was $95.2 \%$ in our study, and this was higher than the rates previously reported. EGFR overexpression has been observed in $29 \%-90 \%$ of esophageal cancers and may be correlated with poor prognosis and response. ${ }^{24}$ EGFR overexpression has also been associated with invasion, metastasis and poor prognosis, ${ }^{8}$ and with chemo- and radio-resistance. ${ }^{25-27}$ In our study, group A patients (EGFR, +++) had a higher OS and MST than group B patients (EGFR, ++). Although the differences between the two groups were insignificant, this finding might be attributable to the small number of patients. Moreover, this result may indicate that the effectiveness of EGFR 
Table 3 Summary of trials of targeted therapy for esophageal cancer

\begin{tabular}{|c|c|c|c|c|c|c|c|}
\hline Year & Authors & Path & NP & NEP & TS & $\mathbf{R R}^{*}$ & M/O \\
\hline 2006 & Enzinger et $\mathrm{a}^{29}$ & $A C$ & 17 & 5 & Cetuximab + Cis/CPT/RT & 13 & \\
\hline 2007 & Safran et $\mathrm{a}^{21}$ & $A C$ & 19 & 19 & Trastuzumab $+\mathrm{Cis} / \mathrm{Pacl} / \mathrm{RT}$ & 43 & $2 y 50$ \\
\hline \multirow[t]{2}{*}{2007} & Dawson et $\mathrm{al}^{30}$ & $A C$ & 10 & 13 & Celecoxib + 5-Fu/Cis/RT & 17 & $19.6 \mathrm{~m}$ \\
\hline & & SCC & 3 & & & & \\
\hline \multirow[t]{2}{*}{2007} & Sunpaweravong et $\mathrm{al}^{31}$ & $A C$ & 34 & 37 & Gefitinib $+5-F u / C i s \pm R T$ & PCR & \\
\hline & & SCC & 3 & & & 25 & \\
\hline 2007 & Pande et $\mathrm{al}^{32}$ & $A C$ & 8 & 8 & Gefitinib + Oxaliplatin/RT & 37.5 & \\
\hline \multirow[t]{2}{*}{2008} & Safran et $\mathrm{al}^{33}$ & $A C$ & 45 & 45 & Cetuximab + Carb/Pacl/RT & 27 & \\
\hline & & SCC & 12 & & & & \\
\hline \multirow[t]{2}{*}{2008} & De Vita et $\mathrm{al}^{34}$ & $A C$ & 9 & 27 & Cetuximab + FOLFOX/RT & 40 & \\
\hline & & SCC & 18 & & & & \\
\hline \multirow[t]{2}{*}{2008} & Rodriguez et $\mathrm{a}^{35}$ & $A C$ & 75 & 28 & Gefitinib + 5-Fu/Cis/RT & & 3y 40 \\
\hline & & SCC & 5 & & & & \\
\hline 2008 & Javle et $\mathrm{al}^{36}$ & $A C$ & 6 & 6 & Gefitinib + Oxaliplatin/RT & 33 & 10.8 \\
\hline \multirow[t]{2}{*}{2009} & Ruhstaller et $\mathrm{al}^{37}$ & $A C$ & 15 & NS & Cetuximab + Cis/Doce/RT & 32 & \\
\hline & & SCC & 13 & & & & \\
\hline 2009 & Ilson et $\mathrm{al}^{38}$ & $A C$ & 18 & 7 & Bevacizumab + Cis/CPT/RT & 10 & \\
\hline \multirow[t]{2}{*}{2009} & Agrawala et $\mathrm{a}^{39}$ & $A C$ & 31 & 20 & Cetuximab + RT & 36 & \\
\hline & & SCC & 9 & & & & \\
\hline 2010 & Li et $\mathrm{al}^{40}$ & SCC & 24 & 24 & Erlotinib $+5-\mathrm{Fu} / \mathrm{Cis} / \mathrm{RT}$ & 87.5 & $2 y 70$ \\
\hline 2012 & Bendell et $\mathrm{al}^{41}$ & $A C$ & 58 & 62 & Bevacizumab + Carb/Pacl/5-Fu/RT & 64 & \\
\hline \multirow[t]{2}{*}{2013} & Crosby et $\mathrm{a}^{42}$ & SCC & 92 & 125 & Cetuximab + Cis/Cap/RT & & $22.1 \mathrm{~m}$ \\
\hline & & $A C$ & 33 & & & & \\
\hline \multirow[t]{2}{*}{2013} & lyer et $\mathrm{al}^{43}$ & $A C$ & 16 & 17 & Erlotinib + RT & & $7.3 \mathrm{~m}$ \\
\hline & & SCC & I & & & & \\
\hline 2013 & Zhai $Y$ et $\mathrm{al}^{44}$ & SCC & 18 & 18 & Erlotinib + RT & 72.2 & $2 y 44.4$ \\
\hline
\end{tabular}

Abbreviations: AC, adenocarcinoma; SCC, squamous cell carcinoma; Carb, carboplatin; Pacl, paclitaxel; RT, radiotherapy; FOLFOX, weekly 5-Fu/oxaliplatin/leucovorin infusion; 5-Fu, 5-fluorouracil; Cis, cisplatin; Doce, docetaxel; CPT, irinotecan; Path, pathology; NP, number of patients; NEP, number of esophageal cancer patients; TS, treatment strategy; RR*, response rate (\%); M/O, median survival time (years/months)/overall survival (\%).

inhibitors increases with increasing levels of EGFR overexpression; however, this inference needs to be confirmed in further studies. The mechanism of combined targeted therapy and radiotherapy most likely includes inhibition of the repair of double-strand breaks in tumor cells, reduction of tumor cell repopulation, blocking of the G1 Phase of the cell cycle in tumor cells, and increase in tumor cell apoptosis. Targeted therapy has been reported to potentiate radiation-induced cell death. ${ }^{28}$ EGFR inhibitors have become the most common targeted-therapy drug that is combined with radiotherapy.

Table 3 summarizes trials of targeted therapy for esophageal cancer. ${ }^{21,29-44}$ The objective clinical response rates in these trials for locally advanced tumors were reported as $10 \%-72 \%$, after excluding the highest rate of $87.5 \%$ that was observed in a trial of Chinese squamous cell carcinoma patients. The MST was 10.8-22 months. The varying pathological types and tumor locations in these trials make a comparison of their results difficult. The most common pathology was adenocarcinoma, which was associated with different malignant transformation-associated genetic lesions rather than those associated with squamous cell carcinoma. The treatment strategies were also different and included targeted therapy alone; combination chemotherapy and targeted therapy; combination radiotherapy and targeted therapy; and combination concurrent chemoradiotherapy and targeted therapy. The drugs used for targeted therapy also differed among these trials. The number of patients in these trials was small. Although some trials showed encouraging results, targeted therapy for esophageal cancer requires further evaluation.

\section{Conclusion}

Although concurrent chemoradiotherapy has been the standard care for locally advanced esophageal cancer, radiotherapy was the choice for those who were refused or could not tolerate chemoradiotherapy. Our study shows that patients with esophageal cancer tolerated nimotuzumab combined with radiotherapy well. The OS after combined h-R3 treatment and radiotherapy was higher than that after historical control radiotherapy alone. EGFR overexpression was common. Further confirmatory studies are required. 


\section{Acknowledgment}

We thank all the nurses, physicists, and technicians who participated in this study.

\section{Disclosure}

The authors of this study declare no conflicts of interest. The sponsor of this study had no role in the study design, data collection, data analysis or data interpretation.

\section{References}

1. Parkin DM, Bray F, Ferlay J, et al. Global cancer statistics, 2002. $C A$ Cancer J Clin. 2005;55(2):74-108.

2. Devesa SS, Blot WJ, Fraumeni JF Jr. Changing patterns in the incidence of esophageal and gastric carcinoma in the United States. Cancer. 1998;83(10):2049-2053.

3. Enzinger PC, Mayer RJ. Esophageal cancer. $N$ Engl J Med. 2003;349(23):2241-2252.

4. Herskovic A, Martz K, al-Sarraf M, et al. Combined chemotherapy and radiotherapy compared with radiotherapy alone in patients with cancer of the esophagus. N Engl J Med. 1992;326(24):1593-1598.

5. Cooper JS, Guo MD, Herskovic A. Chemoradiotherapy of locally advanced esophageal cancer: Long-term follow-up of a prospective radomized trial (RTOG85-01). Radiation Therapy Oncology Group. $J$ Am Med Assoc. 1999;281(17):1623-1627.

6. Al-Sarraf M, Martz K, Herskovic A, et al. Progress report of combined chemotherapy versus radiotherapy alone in patients with esophageal cancer: an intergroup study. J Clin Oncol. 1997;15(1):277-284.

7. Lage A, Crombet T, Gonz'alez G. Targeting epidermal growth factor receptor signaling: early results and future trends in oncology. Ann Med. 2003;35(5):327-336.

8. DobashiY, Stren DF. Membrane-anchored forms of EGF stimulate focus formation and intercellular communication. Oncogene. 1991;6:1151-1159.

9. Pirker R, Pereira JR, Szczesna A, et al; FLEX Study Team. Cetuximab plus chemotherapy in patients with advanced non-small-cell lung cancer (FLEX): an open-label randomized phase III trial. Lancet. 2009;373(9674):1525-1531.

10. Cunningham D, Humblet Y, Siena S, et al. Cetuximab monotherapy and cetuximab plus irinotecan in irinotecan-refractory metastatic colorectal cancer. $N$ Engl J Med. 2004;351(4):337-345.

11. Vermorken JB, Mesia R, Rivera F, et al. Platinum-based chemotherapy plus cetuximab in head and neck cancer. $N$ Engl J Med. 2008;359(11):1116-1127.

12. Crombet-Ramos T, Rak J, Perez R, et al. Antiproliferative, antiangiogenic and proapoptotic activity of h-R3: A humanized anti-EGFR antibody. Int J Cancer. 2002;101(6):567-575.

13. Akashi Y, Okamoto I, Iwasa T, et al. Enhancement of the antitumor activity of ionizing radiation by Nimotuzumab, a humanized monoclonal antibody to the epidermal growth factor receptor, in non-small cell lung cancer cell lines of differing epidermal growth factor receptor status. Br J Cancer. 2008;98(4):749-755.

14. Zhao L, He LR, Xi M, et al. Nimotuzumab promotes radiosensitivity of EGFR-overexpression esophageal squamous cell carcinoma cells by upregulating IGFBP-3. J Transl Med. 2012;10:249

15. Rodríguez MO, Rivero TC, Bai RC, et al. Nimotuzumab plus radiotherapy for unresectable squamous-cell carcinoma of the head and neck. Cancer Biology Therapy. 2010;9(5):343-349.

16. Ramakrishnan MS, Eswaraiah A, Crombet T, et al. Nimotuzumab, a promising therapeutic monoclonal for treatment of tumors of epithelial origin. MAbs. 2009;1(1):41-48.

17. Crombet T, Osorio M, Cruz T, et al. Use of the humanized anti-epidermal growth factor receptor monoclonal antibody h-R3 in combination with radiotherapy in the treatment of locally advanced head and neck cancer patients. J Clin Oncol . 2004;22(9):1646-1654.
18. Common Terminology Criteria for Adverse Events v3.0 (CTCAE). 2006. Available from: http://ctep.cancer.gov/protocolDevelopment/ electronic_applications/docs/ctcaev3.pdf. Accessed September 25, 2013.

19. Eisenhauer E, Therasse P, Bogaerts J, et al. New response evaluation criteria in solid tumours: Revised RECIST guideline (version 1.1). Euro J Cancer. 2009;45(2):228-247.

20. Gotoh M, Takiuchi H, Kawabe S, et al. Epidermal Growth Factor Receptor is a Possible Predictor of Sensitivity to Chemoradiotherapy in the Primary Lesion of Esophageal Squamous Cell Carcinoma. Jpn J Clin Oncol. 2007;37(9):652-657.

21. Safran H, Dipetrillo T, Akerman P, et al. Phase I/II study of transtuzumab paclitaxel, cisplatin and radiation for locally advanced,. HER 2 overexpressing esophageal adenocarcinoma. Int J Radiat Oncol Biol Phys. 2007;67(2):405-409.

22. Han J, Zhu W, Tu C, et al. Clinical study of concurrent chemoradiotherapy or radiotherapy alone for esophageal cancer patients with positive lymph node metastasis. Tumori. 2012;98(1):60-65.

23. Smith TJ, Ryan LM, Douglass HO Jr, et al. Combined chemoradiotherapy vs. radiotherapy alone for early stage squamous cell carcinoma of the esophagus: a study of the Eastern Cooperatve Oncology Group. Int J Radiat Oncol Biol Phys. 1998;42(2):269-276.

24. Kuwano H, Kato H, Miyazaki T, et al. Genetic alterations in esophageal cancer. Surg Today. 2005;35(1):7-18.

25. Sheridan MT, O'Dwyer T, Seymour CB, et al. Potential indicators of radiosensitivity in squamous cell carcinoma of the head and neck. Radiat Oncol Investig. 1997;5(4):180-186.

26. Mason KA, Komaki R, Cox JD, et al. Biology-based combined-modality radiotherapy: workshop report. Int J Radiat Oncol Biol Phys. 2001;50(4):1079-1089.

27. Barker FG II, Simmons ML, Chang SM, et al. EGFR overexpression and radiation response in glioblastoma multiforme. Int J Radiat Oncol Biol Phys. 2001;51(2):410-418.

28. Akimoto T. Molecular basis and clinical results of radiation therapy combined with molecular targeted therapy. Nippon Rinsho. 2010;68:1025-1034.

29. Enzinger P, Yock T, Suh W, et al. Phase II cisplatin, irrinotecan, cetuximab and concurrent radiation therapy followed by surgery for locally advanced esophageal cancer. In: 2006 ASCO Annual Meeting Proceedings (Post-Meeting Edition). J Clin Oncol. 2006;24(Suppl 18): 4064.

30. Dawson SJ, Michael M, Biagi J, et al. A phase I/II trial of celecoxib with chemotherapy and radiotherapy in the treatment of patients with locally advanced oesophageal cancer. Invest New Drugs. 2007;25(2): 123-129.

31. Sunpaweravong P, Sunpaweravong S, Sangthawan D, et al. Combination of gefitinib, cisplatin and 5-Fu chemotherapy, and radiation therapy (RT) in newly diagnosed patients with esophageal carcinoma. In: 2007 ASCO Annual meeting Proceedings (Post-Meeting Edition). J Clin Oncol. 2007;2(18S):4605.

32. Pande AU, Lver RV, RaniA, et al. Epidermal growth factor receptor-directed therapy in esophageal cancer. Oncology. 2007;73(5-6):281-289.

33. Safran H, Suntharalingam M, Dipetrillo T, et al. Cetuximab with concurrent chemoradiation for esophagogastric cancer: assessment of toxicity. Int J Radiat Oncol Biol Phys. 2008;70(2):391-395.

34. De Vita F, Orditura M, Innocente R, et al. Induction primary CT with Folfox-4 and cetuximab followed by RT and cetuximab in locally advanced esophageal cancer (LAEC): Analysis of preliminary data from B152 Trial. In: 2008 ASCO Annual Meeting Proceedings (Post-Meeting Edition). J Clin Oncol. 2008;26(15S):15524.

35. Rodriguez C, Adelstein D, Rybicki L, et al. A phase II trial of perioperative concurrent chemoradiotherapy (CCRT) and gefitinib (G) in locally advanced esophagus (E) and gastroesophageal junction (GEJ) cancer. In: 2008 ASCO Annual Meeting proceedings (Post-Meeting Edition). $J$ Clin Oncol. 2008;26(15S):4547.

36. Javle M, Pande A, Lyer R, et al. Pilot study of gefitinib, oxaliplatin and radiotherapy for esophageal adenocarcinoma: tissue effect predicts clinical response. Am J Clin Oncol. 2008;31(4):329-334. 
37. Ruhstaller T, Pless M, Schuller J, et al; Swiss Group for Clinical Cancer Research (Schweizerische Arbeitsgemeinschaft fur Klinische Krebsforschung) Cetuximab in combination with chemoradiotherapy prior to surgery in patients with resectable, locally advanced esophageal carcinoma: A prospective, multicenter phase I B/II trial (SAKK 75/06). Presented in part at the 45th Annual Meeting of the American Society of Clinical Oncology, May 29-June 2, 2009, Orlando, FL. J Clin Oncol. 2011;29(6):626-631.

38. Ilson D, Bains M, Rizk N, et al. Phase II trial of preoperative bevacizumab (Bev), irinotecan (I), cisplatin (C), and radiation (RT) in esophageal adenocarcinoma: preliminary safety analysis. In: 2009 ASCO Annual Meeting Proceedings (Post-Meeting Edition). J Clin Oncol. 2009;27(15S):4573.

39. Agrawala A, Hanna N, McCollum A, et al. Preoperative cetuximab and radiation (XRT) for patients with surgically resectable esophageal and gastroesophageal junction (GEJ) carcinomas: A pilot study from the Hoosier Oncology Group and the University of Texas Southwestern. In: 2009 ASCO Annual Meeting Proceedings (Post-Meeting Edition). J Clin Oncol. 2009;27(15S):4557.

40. Li G, Hu W, Wang J, et al. Phase II study of concurrent chemoradiation in combination with erlotinib for local advanced esophageal carcinoma. Present in part at the 44th ASCO Annual Meeting, May 30-June, 2008, Chicago, IL. Int J Radiation Oncology Biol Phys. 2010;28(5):1407-1412.
41. Bendell JC, Meluch A, Peyton J, et al. A phase II trial of preoperative concurrent chemotherapy/radiation therapy plus bevacizumab/erlotinib in the treatment of localized esophageal cancer. Clin Adv Hematol Oncol. 2012;10(7):430-437.

42. Crosby T, Hut C, Falk S, et al. Chemoradiotherapy with or without cetuximab in patients with oesophageal cancer (SCOPE1): a multicentre, phase 2/3 randomised trial. Lancet Oncol. 2013;14:627-637.

43. Iyer R, Chhatrala R, Shefter T, et al. Erlotinib and radiation therapy for elderly patients with esophageal cancer- clinical and correlative results from a prospective multicentre phase 2 trial. Oncology. 2013;85:53-58.

44. Zhai Y, Hui Z, Wang J, et al. Concurrent erlotinib and radiotherapy for chemoradiotherapy-intolerant esophageal squamous cell carcinoma patients: results of a pilot study. Dis Esoph. 2013;26:503-509.
OncoTargets and Therapy

\section{Publish your work in this journal}

OncoTargets and Therapy is an international, peer-reviewed, open access journal focusing on the pathological basis of all cancers, potential targets for therapy and treatment protocols employed to improve the management of cancer patients. The journal also focuses on the impact of management programs and new therapeutic agents and protocols on

\section{Dovepress}

patient perspectives such as quality of life, adherence and satisfaction. The manuscript management system is completely online and includes a very quick and fair peer-review system, which is all easy to use. Visit http://www.dovepress.com/testimonials.php to read real quotes from published authors. 